\title{
Dynamic Performance Analysis of the Towers of a Long-Span Bridge Based on GPS Monitoring Technique
}

\author{
Mosbeh R. Kaloop ${ }^{1,2}$ and Jong Wan $\mathrm{Hu}^{1,3}$ \\ ${ }^{1}$ Department of Civil and Environmental Engineering, Incheon National University, 12-1 Songdo-dong, Yeonsu-gu, \\ Incheon 406-840, Republic of Korea \\ ${ }^{2}$ Department of Public Works and Civil Engineering, Mansoura University, Mansoura 35516, Egypt \\ ${ }^{3}$ Incheon Disaster Prevention Research Center, Incheon National University, 12-1 Songdo-dong, Yeonsu-gu, \\ Incheon 406-840, Republic of Korea \\ Correspondence should be addressed to Jong Wan Hu; jongp24@incheon.ac.kr
}

Received 27 August 2015; Revised 14 March 2016; Accepted 23 March 2016

Academic Editor: Christos Riziotis

Copyright (C) 2016 M. R. Kaloop and J. W. Hu. This is an open access article distributed under the Creative Commons Attribution License, which permits unrestricted use, distribution, and reproduction in any medium, provided the original work is properly cited.

\begin{abstract}
The present study investigates the parameter identification and the dynamic performance of a long-span bridge tower based on the output of a global positioning system (GPS) health monitoring system. The random decrement (RD) algorithm is used to estimate the tower displacement impulse response. Three methods are applied to extract the dynamic performance including least squares complex exponential (LSCE) method, Hilbert envelope method (HEM), and eigensystem realization algorithm (ERA). Results reveal that the HEM and LSCE method are more suitable to extract fundamental frequency and modal and damping ratios of the tower. Furthermore, the dynamic properties and statistical time series analysis of the GPS measurements illustrate that the traffic loads have a high significant impact on the semistatic and dynamic performances.
\end{abstract}

\section{Introduction}

Bridges performance has three types of movement components, in the time domain, which are static, semistatic, and dynamic movements' components [1, 2]. The static and semistatic movements of structures are measured based on geodetic or displacement sensors techniques [3, 4]. In addition to conventional terrestrial surveying methods, using robotic total stations and similar surveying equipment, satellite-based positioning techniques have been widely used for monitoring the movement components of structures $[1,5$, 6]. The dynamic component is a main factor to understand the response of structures under dynamic loads $[7,8]$. Furthermore, the accelerometer sensors are extensively used for that purpose. They are installed on a number of points on the bridge girder and tower to estimate the bridge's dynamic performances (natural frequency, mode shapes, and damping ratio), but they are not adequate to precisely estimate the displacement components. The dynamic displacement can be obtained from accelerometer measurements by integrating the measured acceleration data $[9,10]$. The vibration components are high in long-span bridges, and there exist both semistatic displacement and dynamic displacement induced by the dynamic effects $[7,11]$. Therefore, the accelerometers are unable to accurately measure these movements in frequency domain but in time domain they are still limited [9, 10]. In contrast, the geodetic or displacement sensors systems are efficient tools to measure the displacement components but still hold certain limitations to explicitly capture the dynamic performance (damping ratio and natural frequency) of structures. Therefore, the random decrement (RD) algorithm with displacement measurements techniques is mostly used to estimate the dynamic performance of structures in time domain $[10,12]$.

One of the geodetic techniques broadly utilized to measure bridges displacement is global positioning system (GPS) technology [2-4]. Psimoulis et al. [13] and Meng [14] assessed the validity of the GPS application in the dynamic performance studies and found that the GPS provides comprehensive information concerning movement components and 
oscillation frequencies of a wide range of engineering structures. GPS can also be used for identification of their dynamic characteristics and the transient or permanent changes to evaluate the structural health and integrity. Recently, the GPS has become a useful tool for measuring displacement responses of bridges, and the dynamic characteristics can be extracted with the increased GPS sampling frequency [2]. For example, Nakamura [1] has applied a $1 \mathrm{~Hz}$ GPS sampling frequency to measure long-span bridge response due to wind effects and compared it with acceleration measurements and concluded that the GPS is reliable to clarify the semistatic and wind dynamic response behaviors of long-span bridges. In addition, Elnabwy et al. [15] studied the movement components of a steel bridge based on $1 \mathrm{~Hz}$ sampling frequency and found that the GPS can be used to extract the movements' components under low traffic load effects. Furthermore, Moschas and Stiros [2] used a $10 \mathrm{~Hz}$ GPS sampling frequency to extract the short-span bridge performance and revealed the potential of GPS to measure the displacement history and the dynamic performances. Górski [12] used $10 \mathrm{~Hz}$ sampling frequency to measure the dynamic performance of a chimney, and the results proved that the dynamic performance of tall structures can be determined based on GPS measurements.

The methodology of dynamic performance extracted from GPS measurements can be found in $[1,2]$. In addition, the most frequently used methods, which were used earlier, are presented herein: the GPS/accelerometer integration is used widely to improve the GPS extracted dynamic performance $[14,16]$. In addition, the integration and differentiation are widely used to extract the dynamic displacement and acceleration from the accelerometer and GPS measurements, respectively [14]. The double filtration is used to extract the dynamic displacement performance [2]. Furthermore, the fast Fourier transformation is used to extract the natural frequency and the random decrement method is used to identify the damping ratio. On the other hand, the dynamic performance analysis of structures can be studied in time and frequency domains [8]. Many studies have used the frequency domain with acceleration measurements $[2,14]$ and converted the acceleration into displacement to study the dynamic attributes in time domain [16]. In addition, the Enhanced Frequency Domain Decomposition (EFDD) and Stochastic Subspace Identification (SSI) techniques are two well-known identification methods on frequency and time domains $[17,18]$. In addition, the two methods are shown much better with multiple-input data [17]. Nowadays, the GPS-health monitoring can be used directly to assess the structures' performance in time domain, but the major problem regarding GPS applications is the measurement errors. The $\mathrm{RD}$ method is used to extract the impulse response function of structures' displacement. In addition, the estimation of the dynamic structural performances in time domain depends on the impulse response function as concluded by Asmussen [19], Moises [20], and Tanyer [21]. Moises [20] compared between Hilbert envelope method (HEM), complex exponential method (CEM), and the Ibrahim Time Domain (ITD) method, and he found that the HEM gives more reliable damping ratio estimation than the CEM and ITD method in time domain. Tanyer [21] found that the performance of least squares complex exponential (LSCE) method is good with low noise effects in measurement, but when noise is present in the data, this method performs poorly. Moreover, Tanyer [21] stated that the eigensystem realization algorithm (ERA) is more reliable for dynamic performance estimation than the frequency domain methods.

The GPS system was experimentally used on the Tower of Yonghe Bridge to measure the ambient displacement [4]. Furthermore, the bridge deck performance and tower identification system are studied previously based on accelerometer and GPS measurements, respectively [4, 22, 23]. The environmental effects on bridges decks and towers are studied in $[4,22]$. In addition, bridge deck dynamic performance is studied in $[22,24]$. Moreover, the GPS/accelerometer integration for the monitoring of bridge towers is studied in [16]. The relation between bridges towers' dynamic displacement and acceleration response versus external forces (traffic, wind, and temperature) was not clearly addressed in the past. Therefore, this study aims to investigate bridges towers' dynamic performance based on GPS-20 Hz sampling frequency; and the random decrement method during long period monitoring system with external loads effects is applied. In this study, the time domain algorithms are used for the real monitoring data. Three fundamental and reliable algorithms are chosen to estimate the dynamic characteristics of the tower, namely, least squares complex exponential (LSCE) method, Hilbert envelope method (HEM), and eigensystem realization algorithm (ERA). In addition, the tower displacement components are studied in relation with the external loads.

\section{Bridge and Monitoring System Description}

Yonghe Bridge links Tianjin and Hangu cities as a part of highway network in China. The bridge is suspended structure system with two towers. The total span of the bridge is $510.00 \mathrm{~m}$ with a main span of $260.00 \mathrm{~m}$ (Figure 1). The $60.5 \mathrm{~m}$ high towers consist of two transverse beams. More details of the bridge construction materials and properties can be found in [20]. In 2007, the maintenance of the bridge was finished and the bridge reopened. For bridge response monitoring, a long-term structural health monitoring (SHM) system was designed [20]. The SHM system for the bridge comprises a data acquisition and processing system with a total of 179 sensors, including accelerometers, strain gauges, displacement transducers, anemometers, temperature sensors, weight-in-motion sensors, and three GPS receivers (Figures 1 and 2).

The data analyzed in this study are collected using two GPS (rover) receivers installed at the top of two towers and one base station clamped at a bank near the bridge (Figure 2). The GPS observations are real-time kinematic (RTK) with differential GPS (DGPS) system. The receivers are LEICA-GMX902 antenna (24-channel L1/L2 code and phase, $20 \mathrm{~Hz}$ data rate, Smart Track technology for high precession, accuracy of $1 \mathrm{~mm}+0.5 \mathrm{ppm}$ (horz.); $2 \mathrm{~mm}+1 \mathrm{ppm}$ (ver.)) and the GPS data were preprocessed using the software LeicaSpider 2.1 to improve and adjust the collected GPS data 


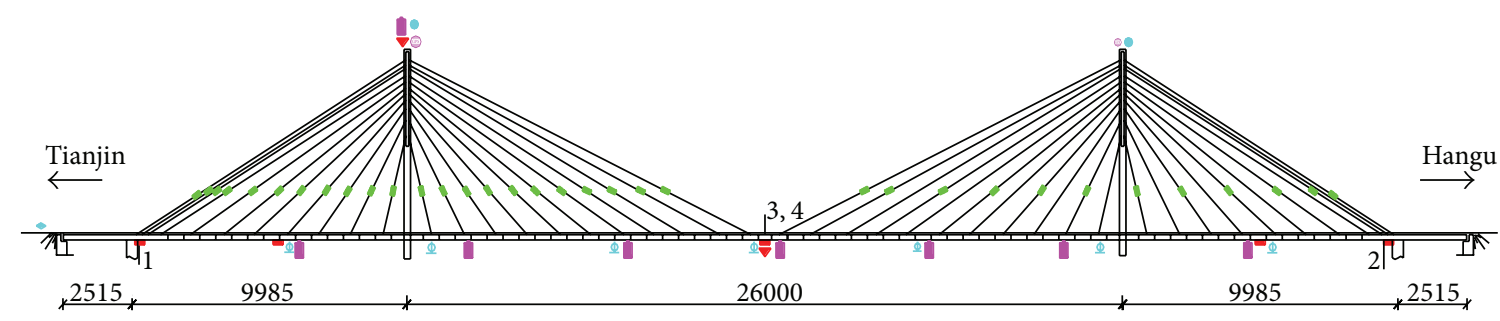

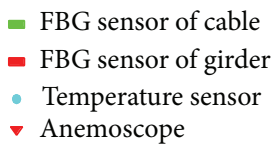

- FBG sensor of cable

- FBG sensor of girder

- Temperature sensor

$\checkmark$ Anemoscope

\author{
- WIM system \\ - Accelerometer \\ $\Phi$ Displacement transducer \\ GPS
}

Figure 1: Bridge elevation and monitoring system (dimensions in $\mathrm{cm}$ ).

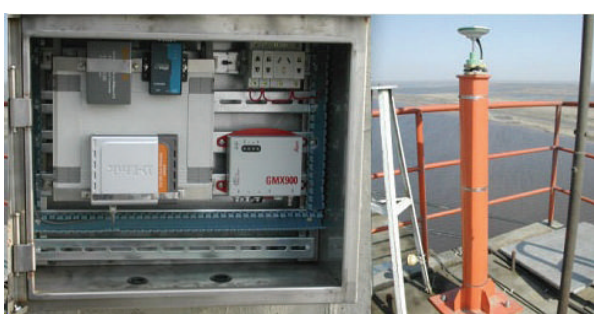

(a)

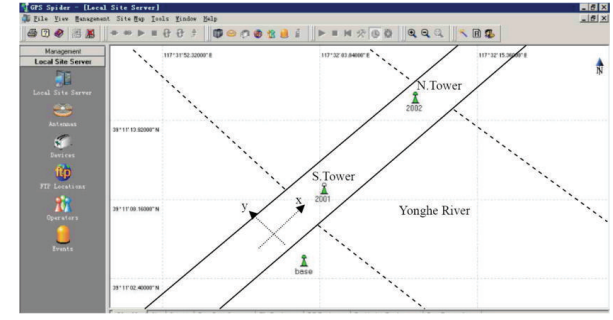

(b)

FIGURE 2: (a) Tower-GPS monitoring and (b) axes definition of the local coordinate system and locations of the GPS monitoring systems.

[25]. The GPS receivers linked via internet to Spider server and raw data is streaming out from receiver to the Spider server. The server generates RINEX-RTK corrections and they are streamed out via TCP/IP to the field. Using LeicaSpider for real-time analysis and error modeling provides consistent high accuracy and improved RTK performance $[4,25]$. Furthermore, the GPS measurements were free of any obstructions of the horizon view and more than 6 satellites were tracked continuously. The coordinate components for each observation epoch are derived. Hence, the time sequences of positions for each station located on the bridge were generated. The output of the two rovers was the time series of global Cartesian coordinates of the installed receivers in the WGS84 coordinate system. Therefore, the collected coordinate's data are converted to a local bridge coordinate system (BCS) for the analysis and evaluation procedures. In this coordinate system, $x$-axis is aligned with the traffic direction; $y$-axis points are aligned towards the lateral direction; and $z$-axis gives the vertical direction of the bridge (Figure 2(b)). The analysis of the movements in this study is focused on the planar coordinates because the $z$-direction movement is not effective in this case. This coordinate system allowed evaluating the performance of the structure and describing the movements of the towers in an appropriate way as it is related to the movement directions of the towers. Herein, the receivers planar coordinates, which we examine in the present paper, consist of a time series of apparent horizontal displacement around a relative zero representing the equilibrium level of the monitoring point.

\section{GPS Measurement Accuracy Assessment}

Zhang et al. [26] estimated the measurement accuracy of monitoring GPS system based on statistical analysis for two days. Therefore, in this study, two days monitoring data, 11 and 12 January 2008, are considered for the analysis of the dynamic performance of the towers. One-minute, fiveminute, ten-minute, and one-hour data with interval of one hour are selected to analyze 24 groups of data. The mean (m) and standard deviation (s) of observation residuals are listed in Table 1.

It can be seen from Table 1 that the accuracy obtained from one and five minutes is better than $\pm 4 \mathrm{~mm}$ in both directions. Compared with the results obtained from ten minutes and one hour, the mean square error decreases with smaller data intervals and this complies with that of Zhang et al. [26]. Therefore, the results indicated that the GPS positioning accuracy is better than $\pm 6 \mathrm{~mm}$; in addition, this accuracy is acceptable to measure the semistatic and dynamic performances of the towers.

\section{Preanalysis Towers Performance}

The displacement components of the tower movements are calculated and discussed in this section. To analyze the towers performance, the GPS observations are first filtered to denoise the time series of GPS receiver's outputs after converting the coordinates into the local bridge coordinate system. Also, to estimate the semistatic movement of 
TABLE 1: Statistical analysis of bridge towers (unit: $\mathrm{m}$ ).

\begin{tabular}{|c|c|c|c|c|c|c|c|c|}
\hline \multirow{2}{*}{ Time groups } & \multicolumn{4}{|c|}{ South tower } & \multicolumn{4}{|c|}{ North tower } \\
\hline & $m_{x}$ & $s_{x}$ & $m_{y}$ & $s_{y}$ & $m_{x}$ & $s_{x}$ & $m_{y}$ & $s_{y}$ \\
\hline $1 \mathrm{~min}$ & $3.465 e-4$ & \pm 0.002 & $6.426 e-4$ & \pm 0.002 & $9.372 e-4$ & \pm 0.003 & $2.059 e-5$ & \pm 0.002 \\
\hline $5 \mathrm{~min}$ & $2.797 e-4$ & \pm 0.003 & $9.143 e-4$ & \pm 0.003 & $6.978 e-4$ & \pm 0.004 & $2.624 e-4$ & \pm 0.003 \\
\hline $10 \mathrm{~min}$ & $2.598 e-4$ & \pm 0.004 & $9.246 e-4$ & \pm 0.003 & $8.853 e-4$ & \pm 0.005 & $5.366 e-4$ & \pm 0.004 \\
\hline $1 \mathrm{~h}$ & $1.540 e-4$ & \pm 0.006 & $1.07 e-4$ & \pm 0.005 & $3.814 e-4$ & \pm 0.006 & $2.822 e-4$ & \pm 0.005 \\
\hline
\end{tabular}

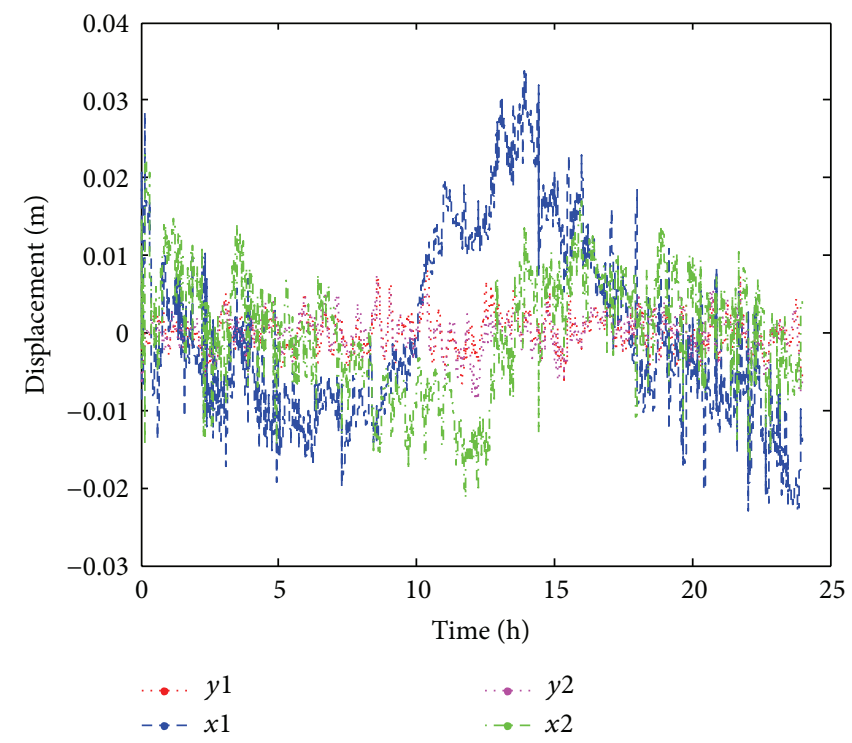

(a)

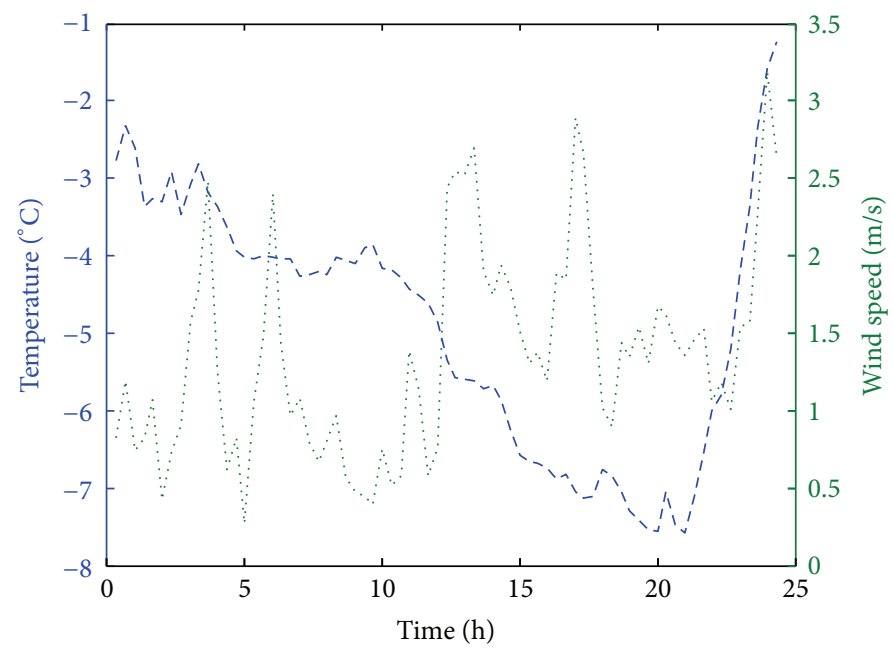

(b)

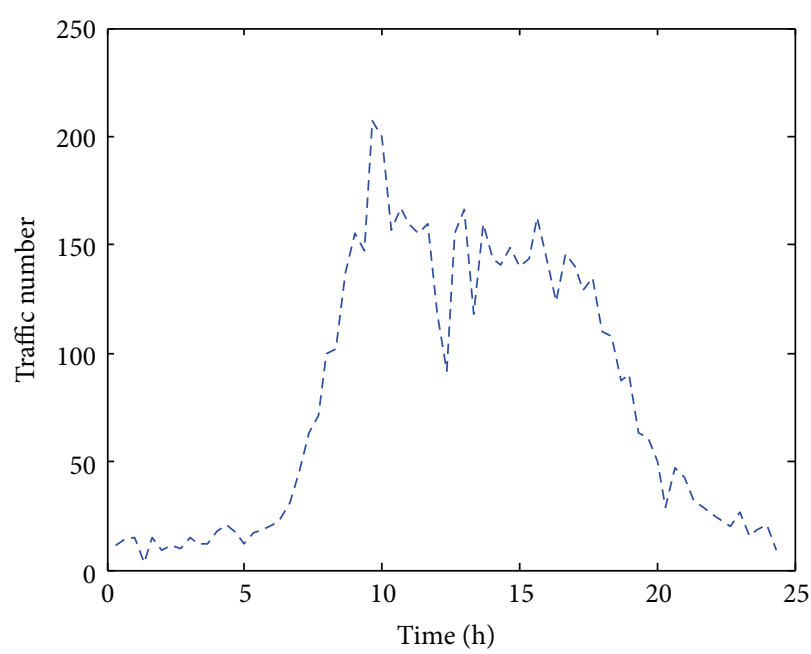

(c)

FIGURE 3: One day (11 January 2008) (a) GPS monitoring data of south tower $(x 1, y 1)$ and north tower $(x 2, y 2)$, (b) temperature (blue) and wind speed (green), and (c) volume of traffic.

components of the towers, the low-pass moving average (MA) filter $(0.025 \mathrm{~Hz})$ is applied to remove the dynamic and noise components from the data. In particular, Moschas and Stiros [2] found that the MA filter is acceptable to classify the long and short period components. The dynamic response can be extracted from the short period components by applying a bandpass filter $[2,15]$. Herein, we used a fourthorder Butterworth filter with bandpass of 0.2 to $0.5 \mathrm{~Hz}$ to extract the dynamic performance of the bridge towers at the first mode shape. The model shapes and frequencies of the Yonghe Bridge are calculated based on finite element analysis as presented in $[16,24]$. Figure 3(a) shows one day 

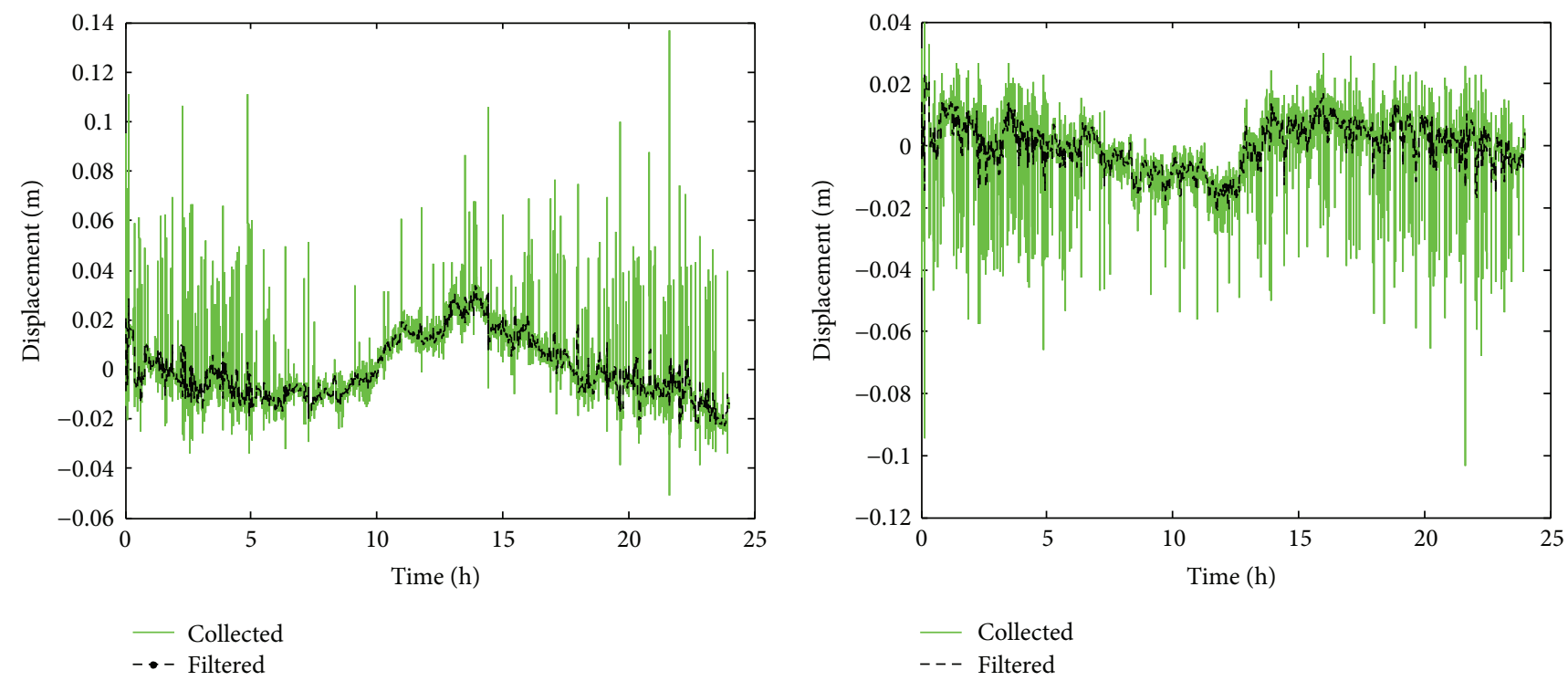

(a)

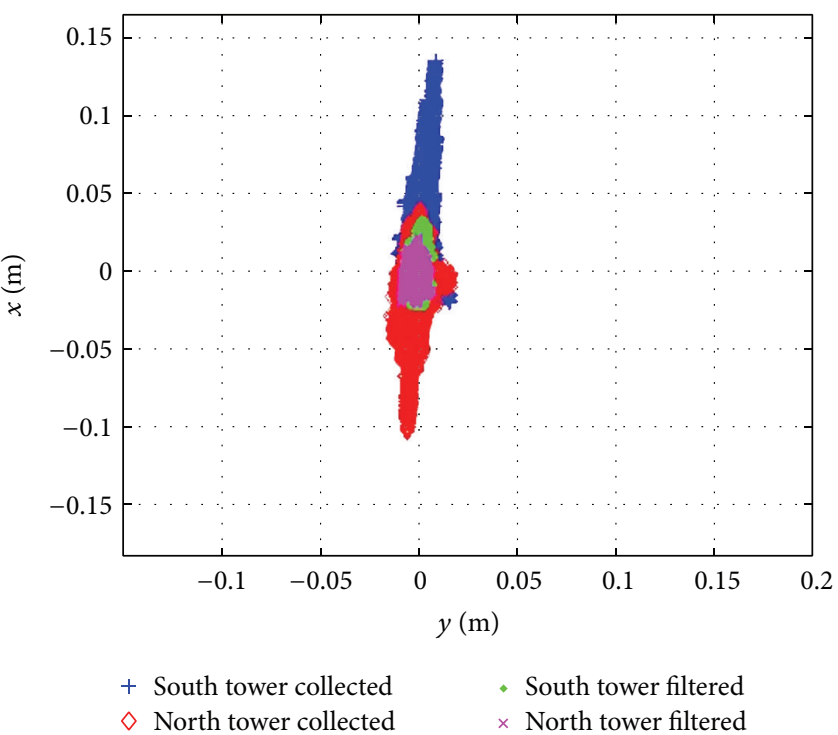

(b)

FIgURE 4: (a) South (left) and north (right) towers collected and filtered for the GPS of $x$-direction measurements. (b) The collected and filtered monitoring data of the two towers.

(11 January 2008) filtration of the towers monitoring data. Figures 3(b) and 3(c) represent the mean of the wind speed, temperature, and traffic numbers per 20 minutes for the same day, respectively. In addition, Figure 4 presents and illustrates the effect of MA filter and the scattered $x$ and $y$ plot of the monitoring data before and after filter application of the north $\left(x_{2}, y_{2}\right)$ and the south $(x 1, y 1)$ towers.

From Figure 3(a), it can be seen that the correlation between the two towers' observations in $x$-direction is noted, except for the time interval from 8:30 to 18:30. In addition, the correlation between $y$-directions of the two towers is higher (0.85) than that of $x$-directions (0.57). Furthermore, the semistatic deformation components (filtered) of the two towers are in the same range of displacement, that is, between 3.22 and $-2.14 \mathrm{~cm}$ in $x$-direction and between 0.75 and $-0.77 \mathrm{~cm}$ in $y$-direction of two towers. In addition, the maximum traffic across the bridge occurred during the period from 8:30 to $18: 30$, whereas the temperature and wind speed have small changes (Figures 3(b) and 3(c)). Accordingly, the traffic load is mainly responsible for the towers' movements.

Figure 4 shows the collected movements for the south tower between 13.68 and $-5.10 \mathrm{~cm}$ and between 1.68 and $-1.08 \mathrm{~cm}$ in $x$-direction and $y$-direction, respectively; meanwhile, for the north tower, the movements are between 4.00 and $-10.32 \mathrm{~cm}$ and between 1.65 and $-1.38 \mathrm{~cm}$ in $x$-direction and $y$-direction, respectively. A combined examination of Figures 3 and 4 reveals that the movements of the two towers in $x$-direction due to the effect of the dynamic load are not 


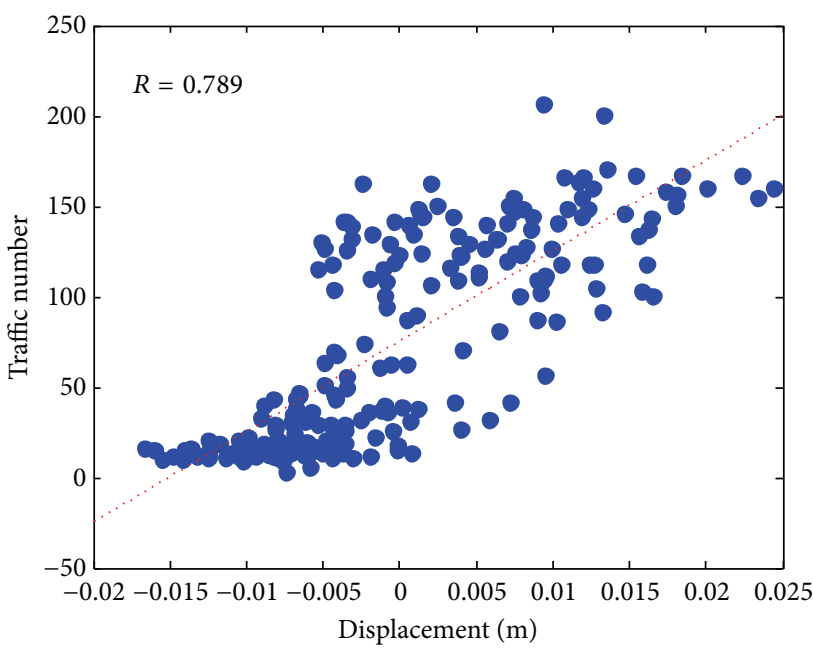

(a)

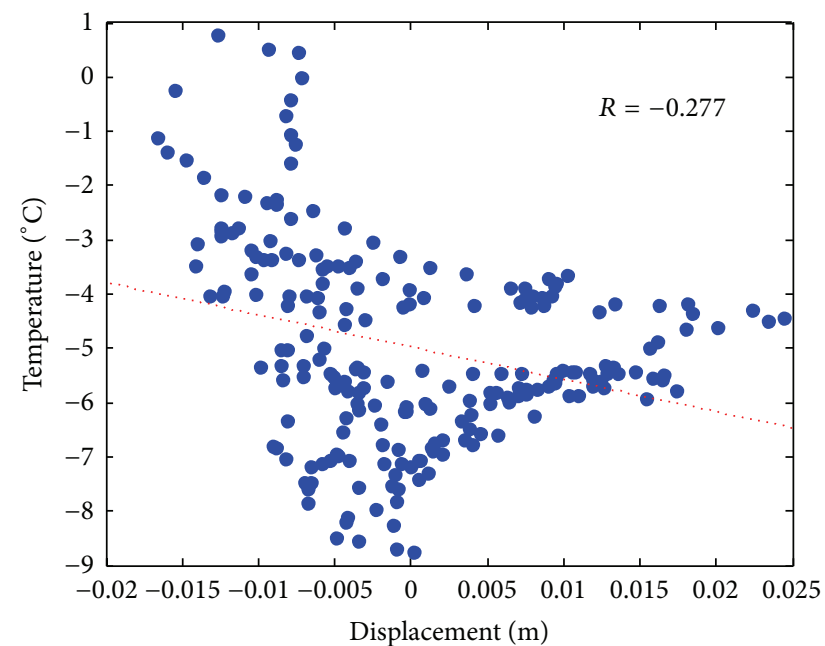

(b)

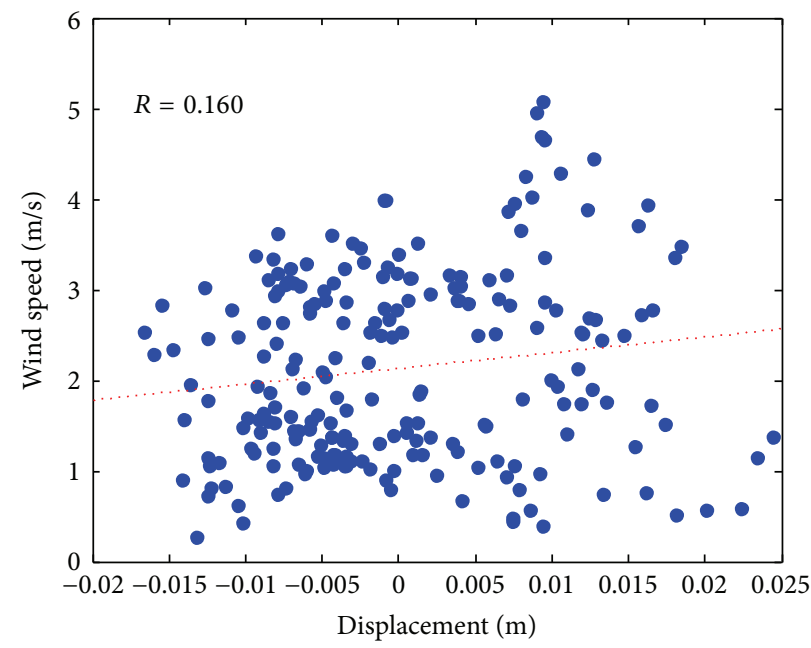

(c)

FIGURE 5: $x$-south tower movements versus average temperature, wind speed, and traffic loads.

symmetrical. From these results, it can be concluded that the dynamic effects are greater than semistatic effects on the towers. In addition, the $x$-direction movements of the towers are greater than the $y$-direction movements in both the semistatic and dynamic performances. In addition, from Figure 4(a), it can be seen that the low-pass MA filter denoises the GPS observations effectively and extracts the semistatic movement components. Moreover, the collected GPS data shows higher displacement with low traffic effects and hence signifies that the dynamic movements of towers are noisy which should be filtered prior to the dynamic performance investigation; thus, the bandpass filter (as shown in Figure 6) is utilized to account for the observed noises. In addition, it can be shown that the semistatic movements in $x$-direction for the south tower are the most efficient, while the north tower movements are shown to be uncorrelated with the traffic loads (see Table 2).

The main causes of the dynamic performance are ambient environmental and traffic loads effects. Li et al. [22]
TABLE 2: Correlation coefficient of ambient environmental and traffic load.

\begin{tabular}{lcccc}
\hline \multirow{2}{*}{ Load } & \multicolumn{2}{c}{ South tower } & \multicolumn{2}{c}{ North tower } \\
& $x$ & $y$ & $x$ & $y$ \\
\hline Traffic & 0.789 & 0.332 & 0.162 & 0.076 \\
Wind speed & 0.160 & 0.169 & 0.403 & 0.302 \\
Temperature & -0.277 & -0.226 & -0.360 & -0.099 \\
\hline
\end{tabular}

represented the relation between the environmental effect and bridge modal parameters and they concluded that the ambient environmental effect is insignificant on the entire bridge's modal shape. Figure 5 represents the relation between the mean 20-minute GPS $x$-direction semistatic movement of the south tower and the traffic numbers, temperature, and wind speed, respectively, for three days from 0:00 on 10 January 2008 to $00: 00$ on 13 January 2008. In addition, the correlation coefficient between loads effects and displacement of the towers movements are listed in Table 2. 


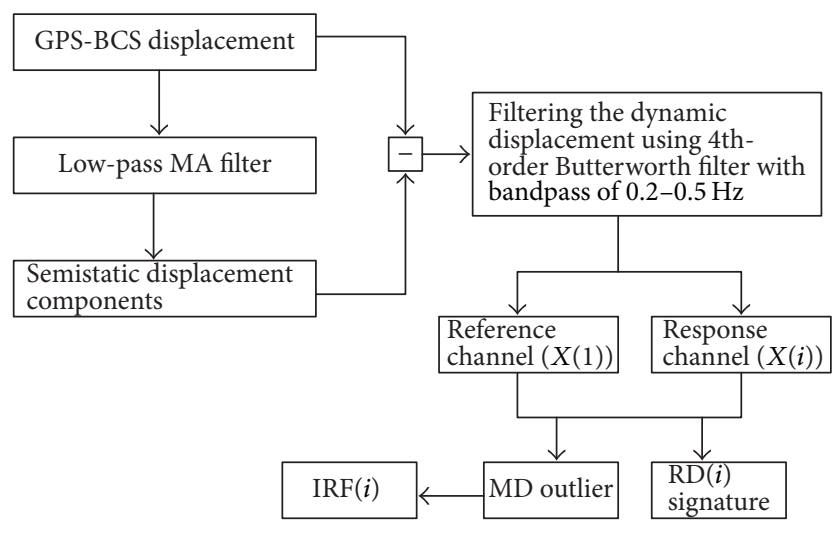

FIGURE 6: Flowchart of RD and IRF extraction from GPSdisplacement measurements.

Using linear fitting, the correlation coefficients between the $x$-direction displacement and traffic, wind speed, and temperature of the south tower are $0.789,0.160$, and -0.277 , respectively, as shown in Figure 5 and Table 2. Furthermore, the maximum volume of traffic is between 10:00 and 17:00 for the three days. Thus this means that the main reason for the south tower movement is the traffic load.

From Table 2, it can be seen that the correlations between $x$-direction and $y$-direction of the south tower and traffic load are positive with high significant values, while the wind speed shows low significant effect. In addition, the temperature effects have a negative correlation with medium significant displacement effect for the two towers. On the other hand, the correlation of the north tower with traffic and wind speed is shown to be puzzled with assumed bridge symmetrical movement's performances with the same loads effects. The north tower's displacement shows a high significant correlation with wind speed and low correlation with traffic load in $x$-direction and $y$-direction movements. Therefore, it can be concluded that the movements of the towers are not symmetrical in $x$-direction and maybe this is the main reason of bridge failure after five months from selection dates on this study [23]. The damage was observed for the closure segment at both side spans and at the support of piers, while the damage appears to be higher near to south tower (refer to Kaloop and Hu [23] and Li et al. [24]). Furthermore, it can be summarized that the traffic and wind loads affected the south and north towers behaviors, respectively, especially in $x$-directions. Moreover, the movements of southern tower are more significant, especially in $x$-direction. Therefore, the dynamic performance in $x$-direction for the south tower will be considered in the next section.

\section{Dynamic Tower Performance Methods and Analysis}

5.1. Theoretical Algorithm for Dynamic Properties Performance. This study aims to use the most common methodology to extract the dynamic performance of the Tower of Yonghe Bridge using GPS monitoring technique. Therefore, this part summarizes the random decrement method with an outlier application and three reliable algorithms to estimate the bridge damping and frequency.

5.1.1. Random Decrement $(R D)$. The random decrement $(\mathrm{RD})$ method has been extensively used for signature analysis of vibrating systems. It is one of the time domain signal processing methods that can be applied in an output-only modal analysis. The RD method can be used if the first natural frequency of a structure is greater than $0.05 \mathrm{~Hz}$. More description of the RD method can be found in [27-29]. The autocorrelation function $\left(R_{x x}\right)$ between time series GPS observations $(x(t))$ for the time window period $(\tau)$ can be presented as follows:

$$
R_{x x}(\tau)=E[x(t+\tau) x(t)],
$$

where $E$ denotes the expected value. If the time lag of $x(t)$ approaches zero, the variance equals the autocorrelation $\left(R_{x x}(0)=\sigma_{x}^{2}\right)$. Vandiver et al. [30] summarized the RD signature $\left(\delta_{x x}(\tau)\right)$ when the excitation function can be treated as a zero mean, stationary, Gaussian, white noise random process:

$$
\delta_{x x}(\tau)=\frac{R_{x x}(\tau)}{\sigma_{x}^{2}} x_{s},
$$

where $x_{s}$ is the threshold level for the acquisition of sample time history $x(t)$ and is generally defined as the root-meansquare value of system's stationary displacement response $x(t)$.

The nonstationary $\mathrm{RD}$ signature between two response channels $\left(X_{1}\right.$ and $\left.X_{2}\right)$ of dynamic displacement components of GPS measurements is developed by Lin and Chiang [27, 28] and can be defined as

$$
\begin{aligned}
\delta_{X_{1} X_{2}}(\tau) & =\frac{E\left[X_{1}(t) X_{2}(t+\tau)\right]}{E\left[X_{1}^{2}(t)\right]} X_{1}(t) \\
& =\frac{R_{X_{1} X_{2}}(\tau)}{\sigma_{X_{1}}^{2}} X_{1}(t),
\end{aligned}
$$

where $X_{1}(t)$ is the reference being chosen as a response channel which contains overall richer frequency information $[27,28]$. The nonstationary cross-RD signature calculated from (3) is in direct proportion to the nonstationary crosscorrelation function under the assumption of Gaussian processes [28]. The ergodic stochastic response for the two response channels is assumed in this study. The extracted dynamic performance of structure from dynamic displacement of the GPS monitoring time series can be obtained using nonstationary RD signature $\left(\delta_{X_{1} X_{2}}(\tau)\right)$ by the damped free oscillation function or impulse response function (IRF) $(a(\tau))$ according to the following equation:

$$
\delta_{X_{1} X_{2}}(\tau)=a(\tau)+m(\tau),
$$

where $m(\tau)$ is the correction function of the approximation of the RD signature. In this study, the Mahalanobis distance (MD) outlier analysis is applied to estimate the correction 
values. The outlier analysis is important for anomaly detection, because the anomalies in measured values may be due to any reason such as noise, operational and environmental variation, or sensor malfunction, and thus will lead $X_{i}(t)$ matrices to exceed the thresholds and results in false data analysis. Outliers often contain useful information especially about the abnormal characteristic of system which impacts the data generation process. The preprocess outlier analysis was carried out to further verify $X_{i}(t)$ data. This process will make the data flawless to be used for the extraction of oscillation modal parameter (damping ratio $(\zeta)$ and natural frequency $\left(\omega_{n}\right)$ ). Figure 6 shows the overview of the method used to extract the IRF of the GPS monitoring displacement response.

5.1.2. Least Squares Complex Exponential (LSCE). The IRF, extracted from the RD signature, of the GPS output data is used to extract the dynamic performance. The LSCE is used to calculate the natural frequency and damping ratio from IRFGPS output. In this case, the time responses are sampled at $2 n+1$ stance as follows [31]:

$$
a_{i}\left(t_{2 n}\right)=\sum_{k=1}^{2 n} \varphi_{i k} V_{k}^{2 n}
$$

where $V_{k}=e^{-\zeta_{k} \omega_{n k} t}$ and $\varphi_{i k}$ are unknown parameters. Prony's method is used to determine the parameters of (5) with real coefficients $(\beta)$ such that the following equation holds [21, 31]:

$$
\beta_{0}+\beta_{1} V_{k}+\beta_{2} V_{k}^{2}+\cdots+\beta_{2 n} V_{k}^{2 n}=0
$$

Then multiply both sides of (5) and (6); and sum them together:

$$
\sum_{j=0}^{2 n} \beta_{j} a_{i}\left(t_{j}\right)=\sum_{j=0}^{2 n} \beta_{j} \sum_{k=1}^{2 n} \varphi_{i k} V_{k}^{j}=\sum_{k=1}^{2 n} \varphi_{i k} \sum_{j=0}^{2 n} \beta_{j} V_{k}^{j}
$$

From the assumption in (5), the term $\sum_{j=0}^{2 n} \beta_{j} a_{i}\left(t_{j}\right)=0$; therefore, with setting $\beta_{2 n}=-1$, this term can be transformed to

$$
\begin{aligned}
& \beta_{0} a(0)+\beta_{1} a(1)+\beta_{2} a(2)+\cdots+\beta_{2 n-1} a(2 n-1) \\
& \quad=a(2 n) .
\end{aligned}
$$

If $4 n$ samples are taken from the IRF, the Hankel matrix can be constructed as follows:

$$
\begin{gathered}
{\left[\begin{array}{cccc}
a(0) & a(1) & \cdots & a(2 n-1) \\
a(1) & a(2) & \cdots & a(2 n) \\
\vdots & \vdots & \vdots & \vdots \\
a(2 n-1) & a(2 n) & \cdots & a(4 n-2)
\end{array}\right]\left[\begin{array}{c}
\beta_{0} \\
\beta_{1} \\
\vdots \\
\beta_{2 n-1}
\end{array}\right]} \\
=\left[\begin{array}{c}
a(2 n) \\
a(2 n+1) \\
\vdots \\
a(4 n-1)
\end{array}\right] .
\end{gathered}
$$

Equation (9) can be rewritten as $H \beta=a$; from this equation, the least square is used to calculate the parameter $\beta$ and $V_{k}$ can be estimated from (6) as follows:

$$
\begin{aligned}
\beta & =\left(H^{T} H\right)^{-1} H^{T} a, \\
w_{n} & =f_{s} \sqrt{\ln V_{k} \ln V_{k}^{*}}, \\
\zeta & =-\frac{\ln V_{k} V_{k}^{*}}{2 w_{n} f_{s}},
\end{aligned}
$$

where $f_{s}$ is the GPS sampling frequency (in our case $f_{s}=$ $1 / 20)$ and $*$ refers to the conjugate transpose of the $V_{k}$ matrix.

5.1.3. Eigensystem Realization Algorithm (ERA). ERA is a minimum-order realization technique and it is an extension of the Ho-Kalman algorithm that uses singular value decomposition technique for denoising [21]. The realization matrices can reproduce system's input-output relationship [31]. ERA begins with the definition of the Markov parameter of a state space model. Consider a discrete time state space model such that

$$
\begin{gathered}
x_{k+1}=A x_{k}+v_{k+1}, \\
y_{k}=C x_{k}+e_{k},
\end{gathered}
$$

where $v_{k+1}$ and $e_{k}$ are the state noise and modeling inaccuracies vectors, respectively, and they are anticipated as zero mean, and $x_{k+1}$ is the time state vector at time instant $k, y_{k}$ is a vector with the sampled outputs, and $A$ and $C$ are the discrete state and output matrix, respectively.

The output $y_{k}$ in this case is the impulse response of system $a(\tau)$. Therefore, the impulse response can be assumed as follows [21]:

$$
a(\tau)= \begin{cases}1 & n=1 \\ C A^{n-1} & n>1\end{cases}
$$

The term of $C A^{n-1}$ is a Markov parameter of state space model. Therefore, to estimate the output parameters, the impulse response can be defined as the following system $[21,31]$ :

$$
Y(k)=C A^{n-1}
$$

where $Y(k)$ has a dimension of $n \times l$ and $l$ is the dimension of output channels. In practice, it is constructed by column wise concatenation of observation vectors resulting from $l$ different outputs channels. The algorithm begins by constructing $r n \times s l$ generalized Hankel matrix:

$$
\begin{aligned}
& H(k-1) \\
& \quad=\left[\begin{array}{cccc}
Y(k) & Y(k+1) & \cdots & Y(k+s) \\
Y(k+1) & Y(k+2) & \cdots & Y(k+s+1) \\
\vdots & \vdots & \vdots & \vdots \\
Y(k+r) & Y(k+r+1) & \cdots & Y(k+r+s)
\end{array}\right] .
\end{aligned}
$$




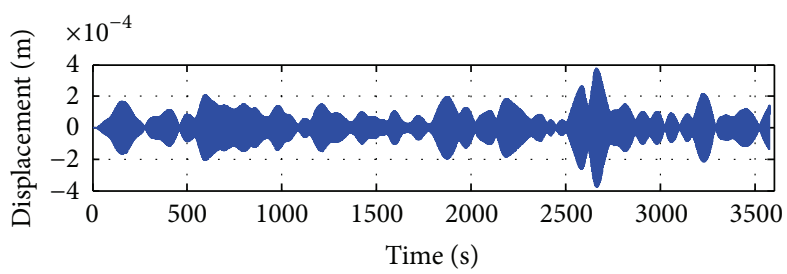

(a)

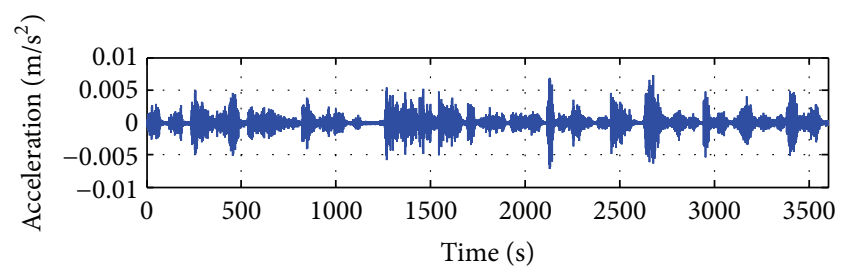

(b)

FIGURE 7: South tower's $x$-direction response: (a) GPS-dynamic displacement; (b) acceleration measurement.

The singular value decomposition of the generalized Hankel matrix $H(0)$ can be calculated as follows:

$$
H=U S V^{T}=\left(U_{1} U_{2}\right)\left(\begin{array}{cc}
S_{1} & 0 \\
0 & S_{2}
\end{array}\right)\left(\begin{array}{c}
V_{1}^{T} \\
V_{2}^{T}
\end{array}\right) \approx U_{1} S_{1} V_{1}^{T} .
$$

The matrix $U$ contains a set of orthonormal "output" basis vector directions for $H$, while $V$ contains a set of orthonormal "input" basis vector directions for $H$. The matrix $S$ contains singular values of the decomposition along its diagonal; here, $S$ is block-separated into two parts, $S_{1}$ and $S_{2}$. The smallest singular values in matrix $S$ are grouped as $S_{2}$ and can be neglected. In contrast, the largest sets of singular values, $S_{1}$, dominate the system and provide a means of assessing the system order [28]. Therefore, the structural discrete state matrix $A$ can be written as follows [31, 32]:

$$
A=\frac{1}{\sqrt{S_{1}}} U_{1}^{T} H_{2 /(i+1)} V_{1} \frac{1}{\sqrt{S_{1}}}
$$

where $H_{2 /(i+1)}$ is Hankel matrix for covariance from 2 to $i+1$. Therefore eigenvalues and eigenvectors can be obtained by the decomposition of the state matrix $A$. Then the modal parameters can be estimated from $A$ matrix as presented in [31-33].

5.1.4. Hilbert Envelope Method (HEM). The IRF $(a(\tau))$ of a single-degree-of-freedom system can be described as follows:

$$
a(t)=A e^{-\zeta \omega_{n} t} \sin \left(\omega_{n}\left(\sqrt{1-\zeta^{2}}\right) t\right)
$$

where $A$ is the residue. Therefore, the analytic signal can be expressed as follows:

$$
\begin{gathered}
\ddot{a}(t)=A e^{-\zeta \omega_{n} t}\left(\sin \left(\omega_{n}\left(\sqrt{1-\zeta^{2}}\right) t\right)\right. \\
\left.+i \cos \left(\omega_{n}\left(\sqrt{1-\zeta^{2}}\right) t\right)\right),
\end{gathered}
$$

where cosine signal was calculated using Hilbert transform technique as shown in [34]. Therefore, the magnitude of the analytic signal eliminates the oscillatory component and gives the envelope as follows:

$$
|\ddot{a}(t)|=A e^{-\zeta \omega_{n} t} .
$$

Taking the natural logarithm of each side yields

$$
\ln |\ddot{a}(t)|=\ln \left(A e^{-\zeta \omega_{n} t}\right)=\ln (A)-\left(\zeta \omega_{n}\right) t .
$$

This is a linear equation. If the slope of the line is calculated, the damping ratio can be estimated as follows:

$$
\zeta=-\frac{\text { slope }}{\omega_{n}}
$$

5.2. Results Finding and Discussions. From the previous analysis, it is concluded that the traffic loads are a main reason of the $x$-direction movements of the south tower. Therefore, in this section, $x$-direction of south tower dynamic response will be studied. Figure 7 shows the south tower response of the dynamic displacement component which is calculated based on Butterworth bandpass filter as shown in Figure 6 and accelerometer acceleration measurement in $x$-direction at 12:00-13:00 on 11 January 2008. This figure illustrates the response of the tower with maximum traffic load effect, whereas at this period the traffic volume passes of the bridge are about 200 vehicles/20 min.

The GPS-dynamic movement components are highly matched with the acceleration response of the tower, as shown in Figure 7. In addition, the maximum response of GPS-dynamic and accelerometer acceleration occurred at $2667 \mathrm{sec}$ for the present monitoring one-hour time period. Furthermore, Figure 8 shows the time frequency of fundamental frequency mode for the same period time selection of the GPS and accelerometer measurements. The frequency is calculated based on Hilbert transform (HT) method [34]. The fourth-order Butterworth bandpass filter of $0.2-$ $2.0 \mathrm{~Hz}$ and $0.2-0.5 \mathrm{~Hz}$ is applied to extract the frequency of the accelerometer and the GPS measurements, respectively, that is, including all frequencies extracted from the GPS measurements $[35,36]$. From Figure 8 , it is seen that the mean fundamental frequency modes of the GPS-dynamic component and acceleration responses are much closer. The mean fundamental frequency of acceleration and GPSdynamic responses are 0.416 and $0.446 \mathrm{~Hz}$, respectively; in addition, the fundamental frequency using the finite element model is $0.418 \mathrm{~Hz}$ [24]. Therefore, the dynamic displacement calculated can be used to detect the dynamic properties of the tower.

The three-day (from 0:00 on 10 January 2008 to 00:00 on 13 January 2008) dynamic properties of the south tower in $x$ direction are represented in this part. The dynamic properties 


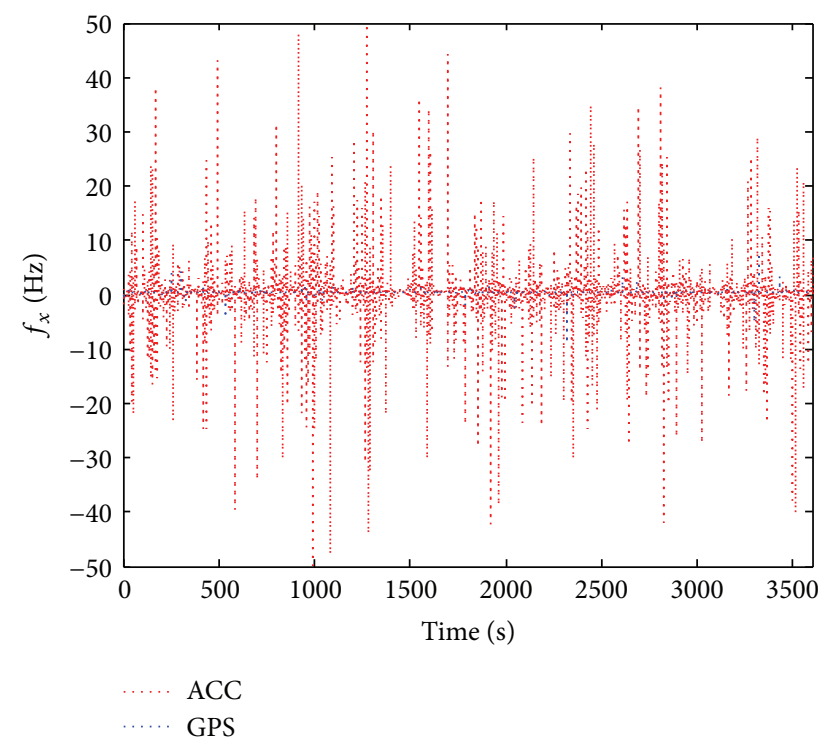

FIGURE 8: Instantaneous frequency of accelerometer and GPS responses of the tower.

of the south tower are calculated based on three methods, presented previously, to extract the frequency and damping ratios. The time interval of the segment can be selected based on Borch's theorem; Li et al. [22] represented this theory. In this theory, the frequency resolution need is a main aim of the time interval required. Therefore, in this study, the frequency resolution is set as $0.0833 \mathrm{~Hz}$ to meet the requirement of GPS-dynamic performance [36]. Therefore, the time interval required is $20 \mathrm{~min}$ for recorded GPS response with sampling frequency of $20 \mathrm{~Hz}$.

Figure 9 illustrates the RD and IRF of the first 20 minutes of Figure 7. In this study, we are using the maximum traffic load time period, which is about 200 vehicles $/ 20 \mathrm{~min}$, as a reference channel, as shown in Figure 6. In addition, Górski [12] recommended the number of time segments to be greater than 900 and time interval of the $\mathrm{RD}$ signature to be greater than $200 \mathrm{~s}$. In this study, we used number of time segments of 4800 and time intervals of $200 \mathrm{~s}$.

The calculated damping ratio from $\mathrm{RD}$ and IRF signatures is 3.22 and $6.02 \%$, respectively, as shown in Figure 9. Li et al. [24] analyzed the bridge models based on finite element and compared the results with the modes identified. The comparative results showed that the absolute differences of the natural frequencies are within 3\% [24], which means that the natural frequency and damping ratio calculations can be used as reference values in this study. The damping ratio of the first vertical model, with fundamental frequency of $0.417 \mathrm{~Hz}$, is found to be $6.54 \%$ [24]. Therefore, the outlier applied is affected to extract the damping ratio within $7.95 \%$. This result concluded that the model design summarized in Figure 6 is affected and can be used to extract the dynamic properties of the tower.

In this study, using only the IRFs, the time domain identification algorithm is adopted to obtain the damped natural frequencies and the damping ratios from the GPS
TABLE 3: Damping ratio and frequency statistical properties of used methods.

\begin{tabular}{lcccccc}
\hline \multirow{2}{*}{ Method } & \multicolumn{3}{c}{ Damping ratio (\%) } & \multicolumn{3}{c}{ Frequency $(\mathrm{Hz})$} \\
& Mean & Range & RC & Mean & Range & RC \\
\hline HEM & 0.060 & 0.005 & 8.43 & 0.420 & 0.023 & 0.767 \\
ERA & 0.055 & 0.166 & 14.77 & 0.444 & 0.414 & 6.546 \\
LSCE & 0.061 & 0.059 & 5.82 & 0.425 & 0.013 & 2.024 \\
\hline
\end{tabular}

data. In addition, the IRF data of the GPS measurements is measured clearly based on the comparison with previous studies. Therefore, the modal parameters of the GPS measurements can be estimated by using the LSCE, ERA, and HEM algorithms based on IRF's calculations. The maximum GPS-dynamic response, at $2667 \mathrm{sec}$, is selected as a reference channel to calculate the IFR for each 20 minutes for the three monitoring days. Herein, we have designed Matlab code based models for each algorithm to calculate the damping ratio and fundamental frequencies for each time segment. LSCE built the Hankel matrix for each time segment (see (9)) and estimated the model parameters using least square solution (see (10)). The time domain IRFs of GPS estimate are utilized as a state space representation in (11) for the ERA method. Therefore, the singular value decomposition of Hankel matrix is applied to find the dynamic performance of the structure (see (14)-(16)). The analytic signal, for the extracted IRF's GPS, of time segments of HEM is calculated as in (18). The magnitude of the analytic signal eliminates the oscillatory component and it is calculated using (19). The slope of the straight line and the frequency calculated by (20) and Hilbert transform, respectively, are used to estimate the damping ratio of the tower.

Figure 10 represents the fundamental frequency and damping ratio of the tower in $x$-direction for the three methods that were presented previously. In addition, Table 3 illustrates the comparison between the three methods used.

From Figure 10(a) and Table 3, it can be seen that the mean damping ratio calculations from the three methods are very close, while the relative change (RC) between mean calculation and $6.54 \%$, which is calculated by Li et al. [24], is shown to have many changes. The worst case method is the ERA method, while the HEM and LSCE method show small relative changes. In addition, the range variance of the HEM is smaller than the ERA and the LSCE method. Furthermore, the relative change of the LSCE method is smaller than the HEM and the ERA method. It means that the HEM and LSCE method are suitable to detect the damping ratios of the extracted IRF-GPS-dynamic performance of the tower. Furthermore, Figure 10(b) and Table 3 show that the relative change between the calculated frequencies and fundamental frequency $(0.417 \mathrm{~Hz}$ [24]) of HEM is smaller than the ERA and the LSCE methods. Furthermore, the three methods can be utilized to estimate the fundamental frequency with different accuracies. The LSCE method shows smaller range variance than the HEM and ERA method. Therefore, the HEM and LSCE method can be used to extract fundamental frequency modal of the GPS-dynamic performance. However, the two methods can be used to 


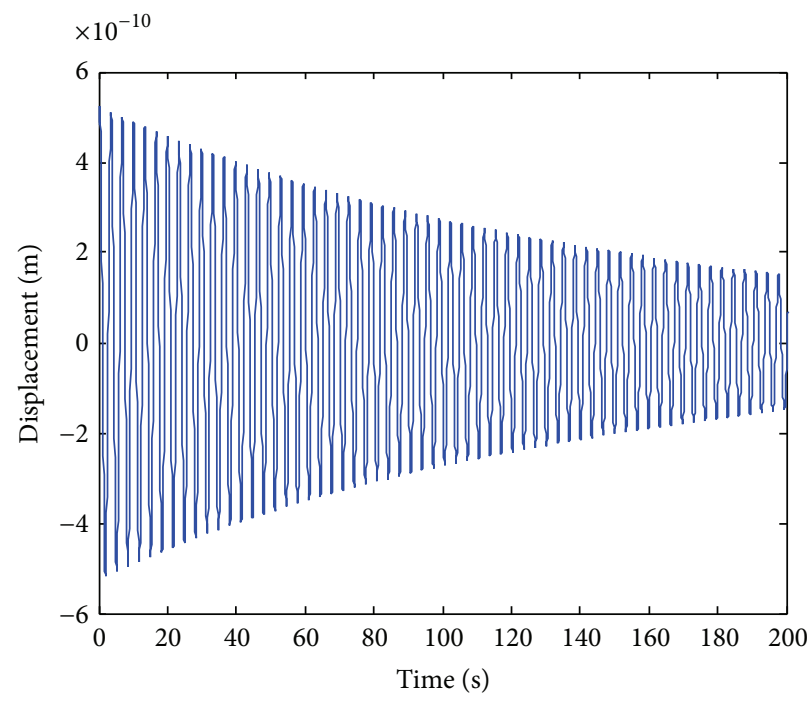

(a)

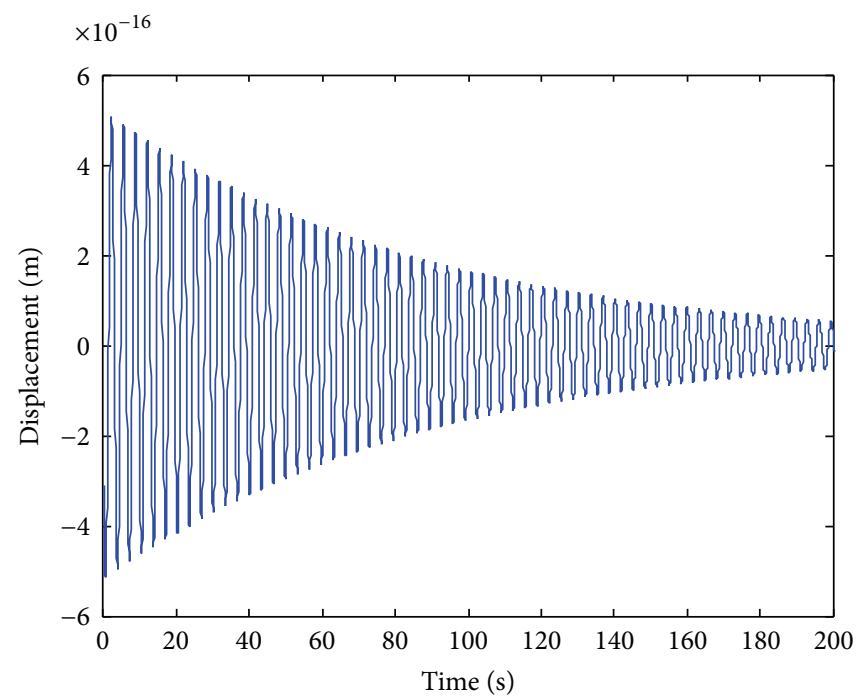

(b)

FIGURE 9: The (a) RD and (b) IRF signature of the tower.

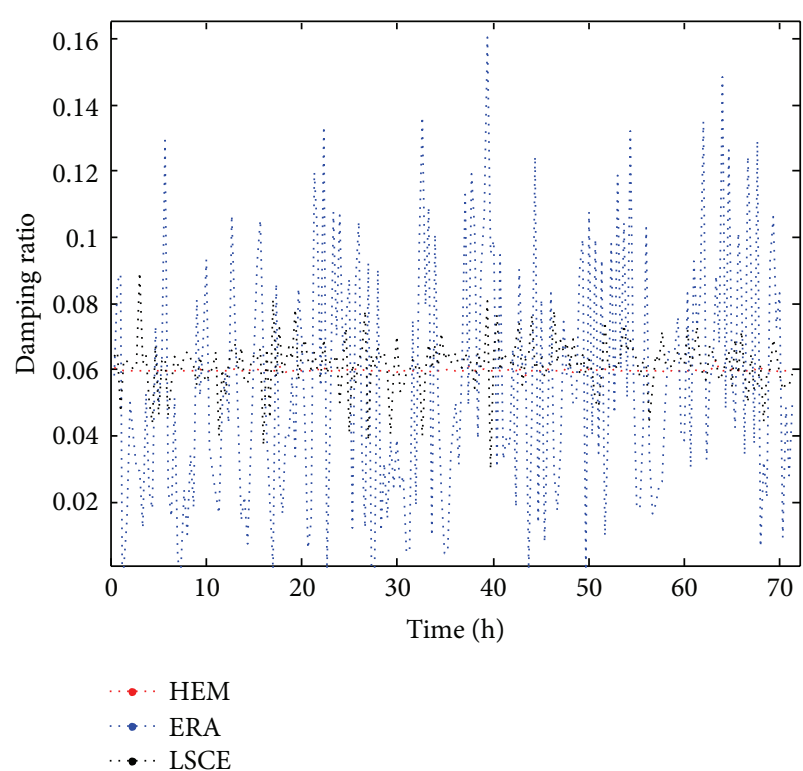

(a)

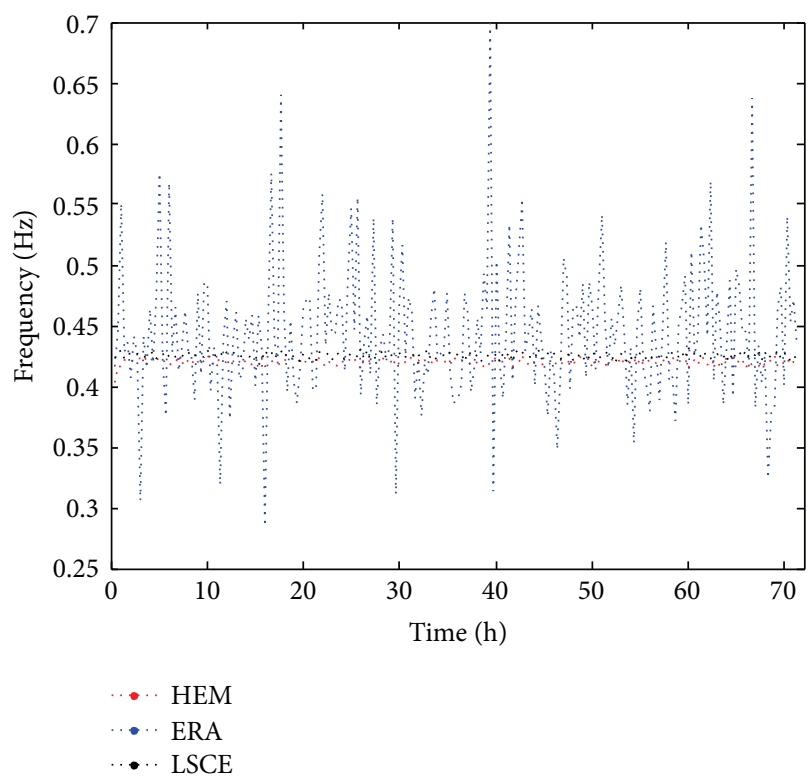

(b)

FIGURE 10: Comparison of three methods used in (a) damping ratio and (b) frequency calculation.

detect the dynamic properties of the tower response. The HEM is easy to use and is reliable and thus was adopted in this study. On the other hand, the results reveal that the GPS-dynamic response contains less effective noises based on Tanyer [21] conclusions, implying that the filters used are appropriate enough to extract the actual response of the tower.

The correlation coefficients between affecting loads and damping ratios and fundamental frequencies calculated based on HEM are listed in Table 4. Figure 11 illustrates the correlation between traffic numbers and HEM damping ratio and fundamental frequencies. The results in Figure 11
TABLE 4: Correlation coefficients of damping ratio and fundamental frequency.

\begin{tabular}{lcc}
\hline Loads & Damping ratio & Frequency \\
\hline Traffic & 0.765 & -0.120 \\
Temperature & -0.0567 & 0.0806 \\
Wind speed & -0.00362 & 0.00883 \\
\hline
\end{tabular}

and Table 4 show that the traffic loads factors have a strong impact on damping ratios and fundamental frequency. Furthermore, the low impact is shown with wind speed and 


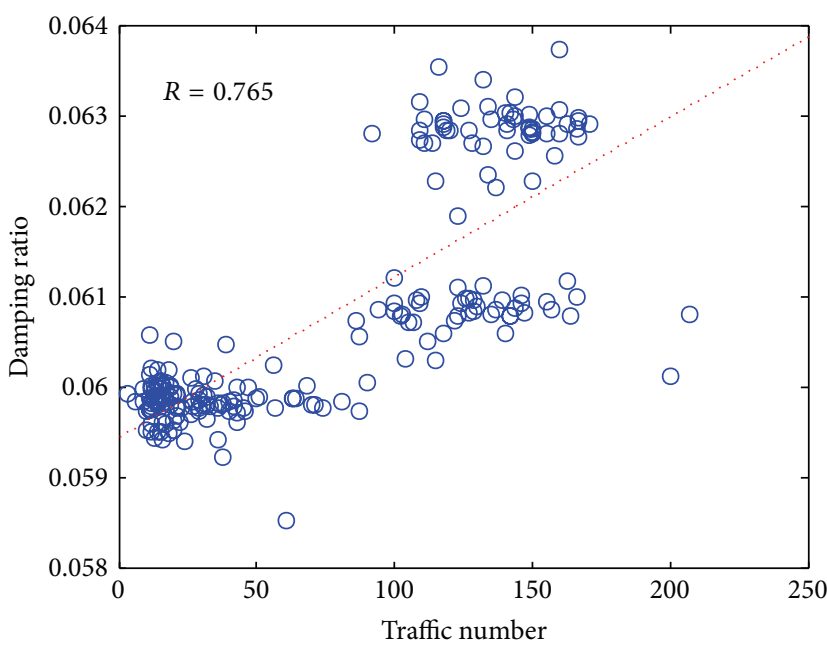

(a)

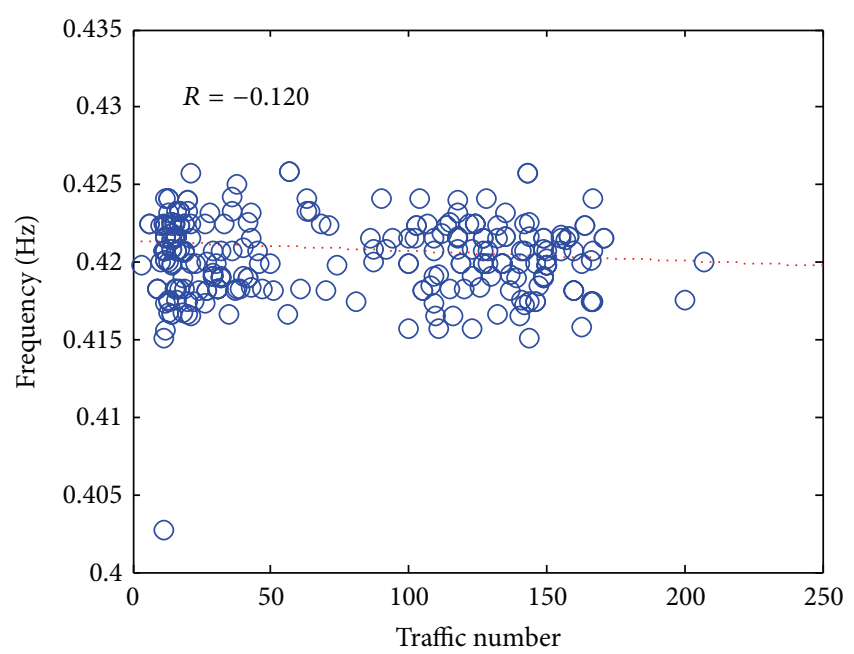

(b)

Figure 11: Traffic volume versus damping ratio and fundamental frequencies.

temperature effects. Therefore, the traffic loads effects have a high significant impact on the dynamic performance of the tower.

Finally, it can be concluded that the tower performance analysis of semistatic and dynamic movements components refers to the effectiveness of moving average and fourthorder Butterworth filters to extract the full tower movements. In addition, the design impulse response based on outlier analysis with HEM and LSCE method is suitable and can be used to detect the dynamic properties of the tower after removing the noises effects. Herein, it should be mentioned that the performance of the LSCE method is adequate when there is no noise effect, while with noise effect in the data this method performs inefficiently [21]; therefore, the applied filter and outlier increased the calculation accuracy of this method. In addition, the ERA incorporates singular value decomposition to counteract inherent noise effects but the Hankel matrix size affected the accuracy of dynamic properties calculation; therefore it gives unsatisfactory results in this study. Meanwhile the HEM seems to be more reliable for dynamic performance estimation than the ERA, when the IRF is truncated using Mahalanobis distance outlier analysis. In addition, the analyzed movements components and loads effects show that the main reason of tower movement and modal identifications is traffic load, while the ambient environmental effects are shown to have insignificant effect.

\section{Conclusions}

Based on the GPS measurements of the tower bridge response to traffic and ambient environmental loads, the fundamental frequency could be extracted first. The previous studies and bridge analysis represent the fundamental frequency and damping ratio of first mode shape which are $0.418 \mathrm{~Hz}$ and $6.54 \%$, respectively. In this study, the moving average filter is applied to extract the semistatic displacement component and moving average with Butterworth bandpass filter which are utilized to detect the dynamic displacement component. The filters' results reveal that the moving average filter is effective to denoise the GPS observations and extracts semistatic movement components; furthermore, the comparison between the calculated GPS-dynamic displacement component and observed acceleration response shows that the two responses are highly matched on the time statistical analysis.

The statistical analysis of semistatic three-day responses of bridge towers with traffic and ambient environmental effects illustrates that the $x$-direction movements of towers are more affected than the $y$-direction movements in semistatic performances. In addition, the significant effect of traffic loads with respect to $x$-direction and $y$-direction of the south tower can be noticed, while the environmental effects have shown lower significance. Furthermore, the correlation of north tower with traffic and wind speed is shown to be puzzled with assumed bridge symmetrical movement's performances with the same load effects. The north tower displacement correlation has shown high significance with wind speed and low significance with traffic loads in $x$ direction and $y$-direction movements. Therefore, it can be concluded that the movements of towers are not symmetrical, especially in $x$-direction, and might be rendered as primary reason of bridge failure after five months from selection dates on this study.

Because of the high sampling frequencies of GPS monitoring systems, the investigation of dynamic characteristic by time domains methods is found to be more suitable in this study. In order to estimate the fundamental frequency and corresponding damping ratios of the tower, the random decrement was applied with Mahalanobis distance outlier analysis. The estimated tower impulse response has shown that the outlier analysis applied is effective to extract the damping ratio within $7.95 \%$. Furthermore, the fundamental frequency and damping ratio calculated are shown to be much closer, compared with previous studies. It infers that 
the model impulse response design can be used to extract the dynamic performance of the bridge.

In this study, least squares complex exponential (LSCE) method, Hilbert envelope method (HEM), and eigensystem realization algorithm (ERA) method are used to detect the damping ratios and fundamental frequency of the south tower in $x$-direction using data of three monitoring days. The comparison between three methods reveals that the HEM and LSCE method are suitable to detect the damping ratios and fundamental frequency of extracted GPS-dynamic performance. In addition, the dynamic properties analysis concludes that the traffic loads have shown a high significant impact on the dynamic performance of the tower, while the ambient environmental effects are quite insignificant.

\section{Competing Interests}

The authors declare that they have no competing interests.

\section{Acknowledgments}

This research was supported by Post-Doctor Research Program in 2015 through the Incheon National University (INU), Incheon, Republic of Korea.

\section{References}

[1] S.-I. Nakamura, "GPS measurement of wind-induced suspension bridge girder displacements," Journal of Structural Engineering, vol. 126, no. 12, pp. 1413-1419, 2000.

[2] F. Moschas and S. Stiros, "Measurement of the dynamic displacements and of the modal frequencies of a short-span pedestrian bridge using GPS and an accelerometer," Engineering Structures, vol. 33, no. 1, pp. 10-17, 2011.

[3] V. Gikas, "Ambient vibration monitoring of slender structuresby microwave interferometer remote sensing," Journal of Applied Geodesy, vol. 6, pp. 167-176, 2012.

[4] M. R. Kaloop and H. Li, "Multi input-single output models identification of tower bridge movements using GPS monitoring system," Measurement, vol. 47, no. 1, pp. 531-539, 2014.

[5] P. A. Psimoulis and S. C. Stiros, "Measuring deflections of a short-span railway bridge using a robotic total station," Journal of Bridge Engineering, vol. 18, no. 2, pp. 182-185, 2013.

[6] V. Gikas, "Smart RTS: monitoring highly dynamic structures," GIM International, vol. 22, no. 6, pp. 44-45, 2008.

[7] C. C. Chang, T. Y. P. Chang, and Q. W. Zhang, "Ambient vibration of long-span cable-stayed bridge," Journal of Bridge Engineering, vol. 6, no. 1, pp. 46-53, 2001.

[8] W.-S. Chan, Y.-L. Xu, X.-L. Ding, Y.-L. Xiong, and W.-J. Dai, "Assessment of dynamic measurement accuracy of GPS in three directions," Journal of Surveying Engineering, vol. 132, no. 3, pp. 108-117, 2006.

[9] C. J. Ku, J. E. Cermak, and L.-S. Chou, "Biased modal estimates from random decrement signatures of forced acceleration responses," Journal of Structural Engineering, vol. 133, no. 8, pp. 1180-1185, 2007.

[10] C. J. Ku, J. E. Cermak, and L.-S. Chou, "Random decrement based method for modal parameter identification of a dynamic system using acceleration responses," Journal of Wind Engineering and Industrial Aerodynamics, vol. 95, no. 6, pp. 389-410, 2007.

[11] M. Zhaobo, Z. Tengfei, and J. Jie, "Dynamic parameters identification for Xi'an bell tower under ambient excitation," in Proceedings of the International Conference on Tissue Engineering (ICTE '11), pp. 1591-1596, American Society of Civil Engineers (ASCE), 2011.

[12] P. Górski, "Investigation of dynamic characteristics of tall industrial chimney based on GPS measurements using Random Decrement Method," Engineering Structures, vol. 83, pp. 30-49, 2015.

[13] P. Psimoulis, S. Pytharouli, D. Karambalis, and S. Stiros, "Potential of Global Positioning System (GPS) to measure frequencies of oscillations of engineering structures," Journal of Sound and Vibration, vol. 318, no. 3, pp. 606-623, 2008.

[14] X. Meng, Real-time deformation monitoring of bridges using GPS/accelerometers [Ph.D. thesis], Institute of Engineering Surveying and Space Geodesy, The Nottingham University, Nottingham, UK, 2002.

[15] M. T. Elnabwy, M. R. Kaloop, and E. Elbeltagi, “Talkha steel highway bridge monitoring and movement identification using RTK-GPS technique," Measurement, vol. 46, no. 10, pp. 42824292, 2013.

[16] M. Kaloop, M. Sayed, and D. Kim, "Movement assessment of a cable-stayed bridge tower based on integrated GPS and accelerometer observations," in Proceedings of the Advanced in Structural Engineering and Mechanics (ASEM '13), pp. 16201630, Jeju, Republic of Korea, September 2013.

[17] A. C. Altunişik, A. Bayraktar, and B. Sevim, "Operational modal analysis of a scaled bridge model using EFDD and SSI methods," Indian Journal of Engineering and Materials Sciences, vol. 19, no. 5, pp. 320-330, 2012.

[18] N.-J. Jacobsen, P. Andersen, and R. Brincker, "Using enhanced frequency domain decomposition as a robust technique to harmonic excitation in operational modal analysis," in Proceedings of the International Conference on Noise and Vibration Engineering (ISMA '06), pp. 3129-3140, Leuven, Belgium, September 2006.

[19] J. Asmussen, Modal Analysis Based on Random Decrement Technique-Application to Civil Engineering Structures, Department of Building and Structural Engineering, University of Aalborg, Aalborg, Denmark, 1997.

[20] A. Moises, Investigating various modal analysis extraction techniques to estimate damping ratio [M.S. thesis], Virginia Polytechnic Institute and State University, Blacksburg, Va, USA, 2000.

[21] I. Tanyer, Parameter estimation for linear dynamical systems with applications to experimental modal analysis [M.S. thesis], Izmir Institute of Technology, Izmir, Turkey, 2008.

[22] H. Li, S. Li, J. Ou, and H. Li, "Modal identification of bridges under varying environmental conditions: temperature and wind effects," Structural Control and Health Monitoring, vol. 17, no. 5, pp. 495-512, 2010.

[23] M. R. Kaloop and J. W. Hu, "Stayed-cable bridge damage detection and localization based on accelerometer health monitoring measurements," Shock and Vibration, vol. 2015, Article ID 102680, 11 pages, 2015.

[24] S. Li, H. Li, Y. Liu, C. Lan, W. Zhou, and J. Ou, "SMC structural health monitoring benchmark problem using monitored data from an actual cable-stayed bridge," Structural Control and Health Monitoring, vol. 21, no. 2, pp. 156-172, 2014. 
[25] M. Kaloop, Structural health monitoring through dynamic and geometric characteristics of bridges extracted from GPS measurements [Ph.D. thesis], Department of Disaster Prevention and Reduction Engineering and Protective Engineering, Harbin Institute of Technology, Harbin, China, 2010.

[26] H. Zhang, B. Yang, and S. Huang, "Dynamic geometry monitoring system and its application in Sutong Bridge construction," Geo-Spatial Information Science, vol. 13, no. 2, pp. 137-143, 2010.

[27] C.-S. Lin and D.-Y. Chiang, "A modified random decrement technique for modal identification from nonstationary ambient response data only," Journal of Mechanical Science and Technology, vol. 26, no. 6, pp. 1687-1696, 2012.

[28] C.-S. Lin and D.-Y. Chiang, "Modal identification from nonstationary ambient response data using extended random decrement algorithm," Computers and Structures, vol. 119, pp. 104-114, 2013.

[29] S. V. Modak, "Separation of structural modes and harmonic frequencies in operational modal Analysis using random decrement," Mechanical Systems and Signal Processing, vol. 41, no. 1-2, pp. 366-379, 2013.

[30] J. K. Vandiver, A. B. Dunwoody, R. B. Campbell, and M. F. Cook, "A mathematical basis forthe random decrement vibration signature analysis technique," Journal of Mechanical Design, vol. 104, no. 2, pp. 307-313, 1982.

[31] W. Zhou and D. Chelidze, "Generalized eigenvalue decomposition in time domain modal parameter identification," Journal of Vibration and Acoustics, vol. 130, no. 1, Article ID 011001, 2008.

[32] J.-H. Weng, C.-H. Loh, J. P. Lynch, K.-C. Lu, P.-Y. Lin, and Y. Wang, "Output-only modal identification of a cable-stayed bridge using wireless monitoring systems," Engineering Structures, vol. 30, no. 7, pp. 1820-1830, 2008.

[33] I. Khan, D. Shan, and K. Malik, "Covariance driven subspace identification technique for continuous modal parameters identification of Sutong Bridge," in Proceedings of the 37th IABSE Symposium on Engineering for Progress, Nature and People, vol. 8, pp. 2666-2673, Madrid, Spain, September 2014.

[34] K. Shin and J. Hammond, Fundamentals of Signal Processing for Sound and Vibration Engineers, John Wiley \& Sons, England, UK, 2008.

[35] M. R. Kaloop and H. Li, “Tower bridge movement analysis with GPS and accelerometer techniques: case study Yonghe tower bridge," Information Technology Journal, vol. 8, no. 8, pp. 12131220, 2009.

[36] M. R. Kaloop and H. Li, "Sensitivity and analysis GPS signals based bridge damage using GPS observations and wavelet transform," Measurement, vol. 44, no. 5, pp. 927-937, 2011. 


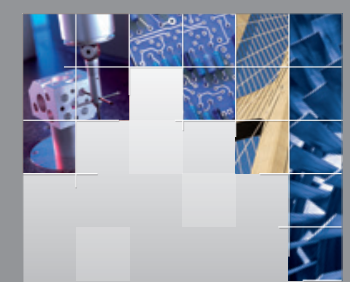

\section{Enfincering}
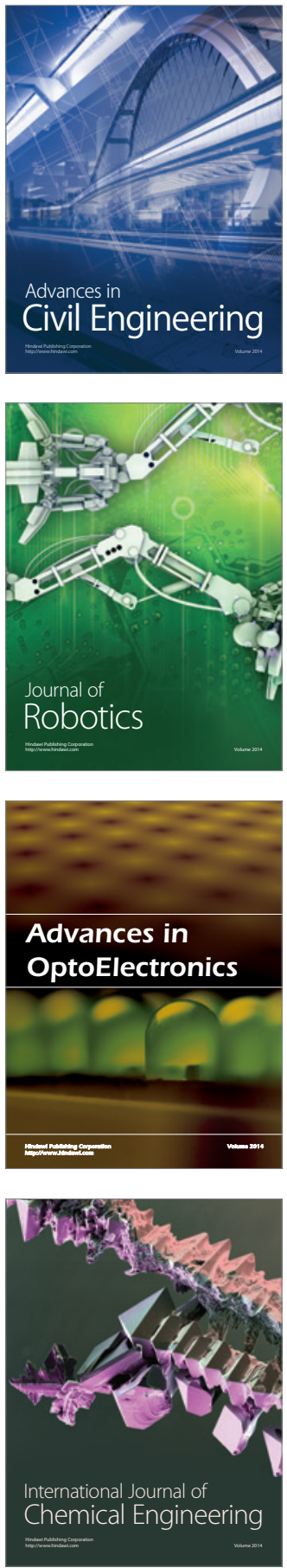

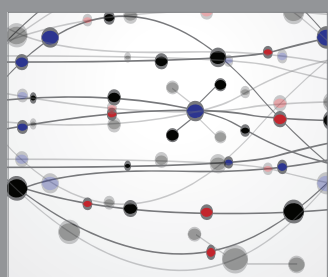

The Scientific World Journal

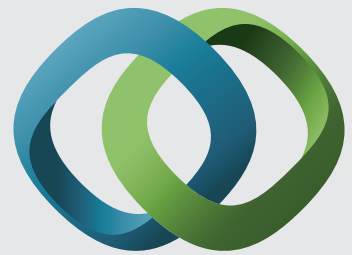

\section{Hindawi}

Submit your manuscripts at

http://www.hindawi.com
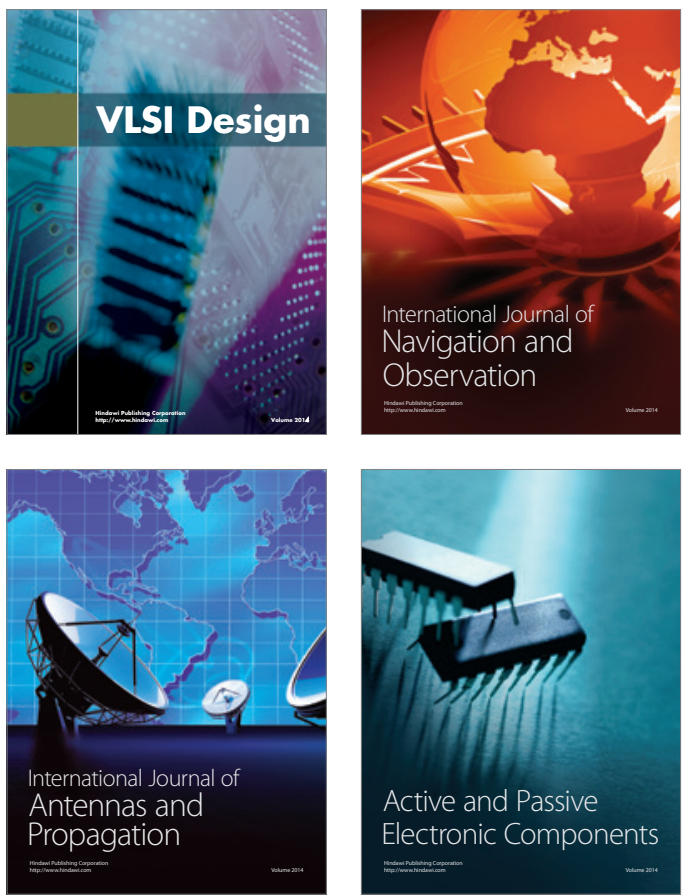
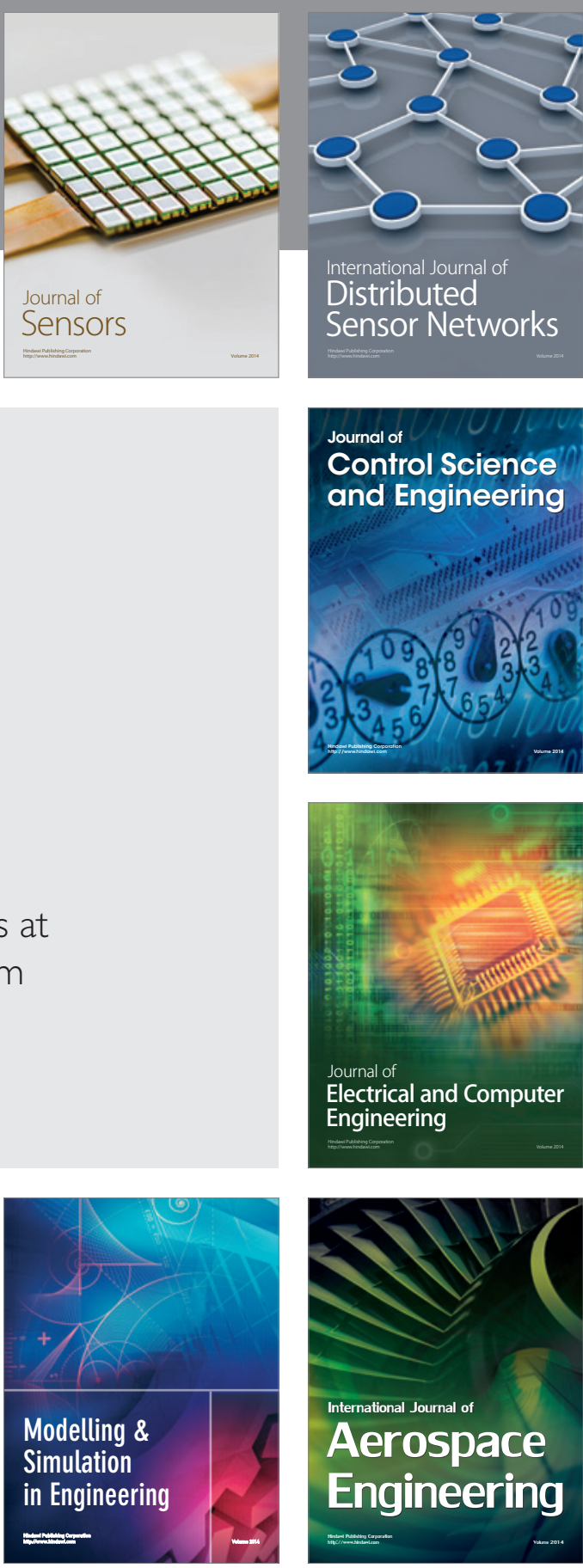

International Journal of

Distributed

Sensor Networks

Journal of

Control Science

and Engineering
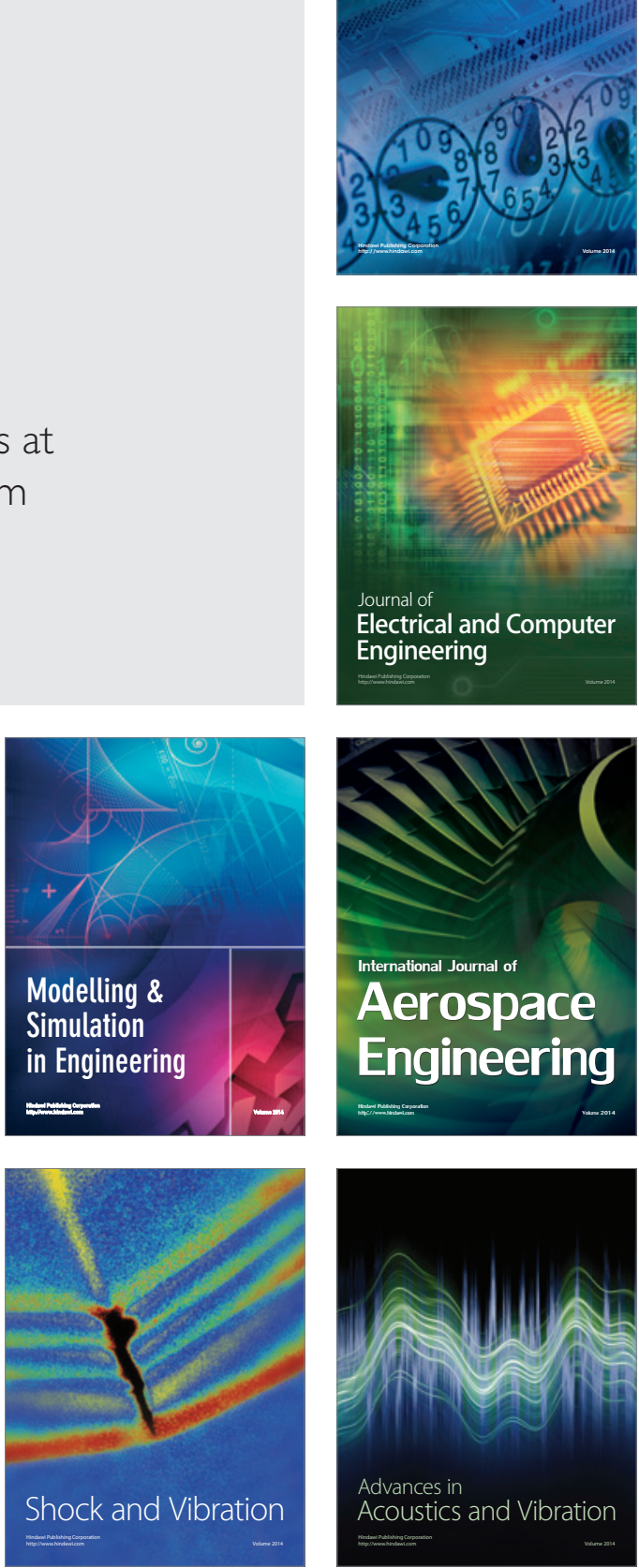